\title{
The Mind/Brain Problem and Perception in Terms of Discrete Quantum Mechanics (DQM)
}

\author{
Kullervo Rainio \\ Helsinki University \\ Suursuontie 29, as. 327, FIN, Helsinki, 00630, Finland \\ Tel: 358-9-7248989_E-mail: kullervo.rainio@pp.inet.fi
}

$\begin{array}{lcc}\text { Received: August 13, } 2011 & \text { Accepted: September 26, } 2011 & \text { Published: December 1, } 2011 \\ \text { doi:10.5539/ijps.v3n2p87 } & \text { URL: http://dx.doi.org/10.5539/ijps.v3n2p87 }\end{array}$

\begin{abstract}
This article is interdisciplinary. It aims to show that we are able, on the basis of discrete quantum mechanics (DQM), to build mathematical models not only for quantum evolution processes but also for processes of mind systems and, particularly, for their interaction. Thus, the solution of the mind/brain problem that Eccles has proposed in his theory of exocytosis is treated by giving it a mathematical form. The key assumption is that quantum systems interact through vector interferences. Eccles' theory is enlarged and made more exact using a discrete quantum mechanical model for the perception. This is also applied to the gestalt structuring process; in particular, the process concerning the gestalt forming of certain reversible (ambiguous) figures is analyzed and the model compared to empirical data.
\end{abstract}

Keywords: Attention, Dendron, Exocytosis, Gestalt, Mind/brain problem, Reversible figure, Transition probability, Vector interference

\section{Introduction}

The problem of consciousness is a controversial issue in modern science. The scientists representing the mainstream in the research, particularly the specialists in brain physiology, are building their ideas on a totally materialistic basis - or trying to eliminate the whole question, claiming that it is unscientific. Most feel themselves forced to approve the existence of consciousness - because they are conscious about their own consciousness - but are of the opinion that it can only be studied from the first person aspect, not from the aspect of the third person, "objectively", as science requires.

In recent years, however, the idea of using quantum mechanics as the frame of reference in the scientific and philosophical analysis of consciousness has become more and more accepted. The focus of the quantum mechanical research into consciousness has so far been in clarifying the interaction between consciousness and matter, i.e., the $\mathrm{mind} /$ body problem:

How is it possible that the mind (self, consciousness,) which is something immaterial, can produce changes in the brain, which is something material - and in that way also in the other parts of the material organism? The other side of the problem - how the stimulation of the senses, something material - can produce mental effects or perceptions, has not been seen as problematic at all, because the reason is clearly material and the consequence has been assumed to be "somehow material", too, when seen from a materialistic viewpoint.

The mind/brain problem is common for physicists, as well as for psychologists, although the orthodox physicists seem to dominate the thinking. They are seriously trying to find a "Theory of Everything" (ToE) but seem to leave the whole problem of consciousness out of it. In mental phenomena they see only "emergent qualities" of matter. It is time to make a serious attempt from the psychological point of view - an attempt to build an exact mathematical framework for explaining the causal interaction between the mind (mental world) and the brain (matter) and vice versa. It is the aim of this article, and is indeed rather ambitious, but quantum mechanics seems to provide the theoretical instrument that is needed.

The quantum mechanical model which is presented in this article is not in accordance with the mainstream scientific paradigm but requires a great change to it, the abandonment of a strictly materialistic viewpoint. The quantum mechanical presentation used in this article, disrete quantum mechanics, is rather far from the mainstream research, at least for the time being. So far quantum mechanics has been based on wave mechanics 
built on the Schrödinger equation, although the discrete point of view - built on Heisenberg's thinking - is closer to the essence of the quantized world.

Discrete quantum mechanics (DQM) is, however, coming. It already has strong supporters. Stanley Gudder, among others, writes in the abstract of his article (Gudder, 1986): "A discrete model for quantum mechanics is presented. First a discrete phase space $\mathrm{S}$ is formed by coupling [the] vertices and edges of a graph. The dynamics is developed by introducing paths or discrete trajectories in S. An amplitude function is used to compute probabilities of quantum events and a discrete Feynman path integral is presented. Many of the results can be formulated in terms of transition probabilities and unitary operators on a Hilbert space."(Italics supplied.)

Atmanspacher describes several weaknesses which he has found in many studies of "quantum consciousness" (Atmanspacher, 2004): "There are quite a number of accounts discussing quantum theory in relation to consciousness that adopt basic ideas of quantum theory in a purely metaphorical manner. Quantum theoretical terms such as entanglement, superposition, collapse, complementarity, and others are used without specific reference to how they are defined precisely and how they are applicable to specific situations."... "But unless such detailed work leads beyond vague metaphors and analogies, they do not yet represent scientific progress." (p. 7).

In this article we try to avoid "vague metaphors and analogies". The concepts used are made mathematically exact and we have tried to show illustratively how they could be applied in specific problem situations, although there is a lack of experimental material, so that the present analysis of consciousness also seems to remain an intermediate form of so-called "thought experiments" and of "vision".

The first part of the article studies what kinds of possible solutions of the psychophysical problem our discrete quantum mechanical frame of reference could open up. In the second part, a model for the (mathematical) structure of consciousness is presented.

\section{The Mind/Brain Problem}

\subsection{The Brain and Consciousness: The Discrete Process Model (DPM)}

Throughout the whole article, Eccles' theory of exocytosis will be used as an example of mind/brain interaction because it is the most detailed and the most illustrative in its concreteness, as Atmanspacher notes (Atmanspacher, 2004): "Probably the most concrete and detailed suggestion of how quantum mechanics can play a role in brain processes is due to Beck and Eccles (1992), later refined by Beck (2001). It refers to particular mechanisms of information transfer at the synaptic cleft. However, ways in which these quantum processes might be relevant for mental activity, and in which their interactions with mental states are conceived, have hardly been elaborated so far." (p. 10). Further: "as indicated above, the proposal outlined so far is the most empirically concrete and theoretically detailed approach to treating brain processes from a quantum theoretical point of view." (p. 10).

Atmanspacher concentrates his criticism on the fact that Eccles does not describe in detail how the dynamics of the large neural wholeness can grow out of the action of separate individual synapses. Atmanspacher also considers that the mental causation in Eccles' presentation remains a mere statement: "The approach by Beck and Eccles is most detailed and concrete with respect to the application of standard quantum mechanics to the process of exocytosis. However, it does not solve the problem of how the activity of single synapses enters the dynamics of neural assemblies, and it leaves mental causation of quantum processes as a mere claim." (Atmanspacher 2004, p. 15). By means of mathematical analysis this article tries to revise some of these weaknesses.

The use of Eccles' theory as an example does not, however, mean that this study is so dependent on it that it would totally lose its significance if that theory were disproved. The application of $D Q M$ which is used in this article is called a discrete process model (DPM). There are some publications which describe it in detail (see, for example, Rainio 2008, 2009a, and 2009b).

\section{DPM in a nutshell:}

The description of the quantum mechanical systems in the DPM is based on the principles of Heisenberg's matrix mechanics.

1. The states of systems are superpositions consisting of discrete element states separable from each other.

2. Time is also a discrete variable, i.e., it moves according to discrete time-steps following each other.

3. Systems move from one state to another during one time-step and follow certain transition probabilities. 
4. At the moment $t$, the transition probabilities from state a to other states constitute the state vector of the state a at the moment $t$. (Remaining in the state a is one possibility and its probability is $p_{a, a}$, included in the state vector.)

A transition probability matrix and a state vector are given as examples in matrix and vector form as follows and in the forms of graphs in Fig. 1. (The number of states $n$ is 4. It could theoretically be $1<n<\infty$.)

A) Transition probability matrix, an example, the same as in Fig. 1:

\begin{tabular}{cccccc}
\multicolumn{5}{c}{ States } & Sum \\
& $\mathrm{s} 1$ & $\mathrm{~s} 2$ & $\mathrm{~s} 3$ & $\mathrm{~s} 4$ & $\Sigma$ \\
$\mathrm{s} 1$ & $\lceil .2$ & .1 & .3 & $.4\rceil$ & 1 \\
$\mathrm{~s} 2$ & .1 & .6 & .1 & .2 & 1 \\
$\mathrm{~s} 3$ & .2 & .1 & .5 & .2 & 1 \\
$\mathrm{~s} 4$ & $\lfloor .3$ & .2 & .1 & $.4\rfloor$ & 1
\end{tabular}

B) State vector:

\begin{tabular}{cccccc}
\multicolumn{5}{c}{ States } & Sum \\
& $\mathrm{s} 1$ & $\mathrm{~s} 2$ & $\mathrm{~s} 3$ & $\mathrm{~s} 4$ & $\Sigma$ \\
$\mathrm{s} 1$ & $(.2$ & .1 & .3 & $.4)$ & 1
\end{tabular}

5. The evolution process of a system is a chain of transitions from one superposition state to another during the time-steps following each other.

6. At every moment, one of the element states of the state vector will be (at the next moment) the actualized state of the system. Which element state it will be is determined as an outcome of a "drawing of lots" according to the transition probabilities.

(As mentioned above, remaining in the actual existing state is also possible.)

Note: According to the DPM model, a so-called "quantum jump" occurs at every time-step. Thus, the highly debated question of how the "quantum measurement" produces the quantum jump is meaningless in this context. The jump is an unavoidable consequence of the discreteness.

7. If the probability of remaining in a certain state $a$, i.e., the probability $\mathrm{p}_{\mathrm{a}, \mathrm{a}}$, is 1 and, thus, the other transition probabilities in the state vector a are 0 , the vector is called a stabilizing state vector. If the evolution process happens to fall on this state $a$ it remains in it.

What is written above represents a condensed account of the system's quantum states and a brief description of their changes, i.e., the transition (evolution) dynamics of quantum systems.

The entanglement of the quantum systems produces its own interference dynamics, which, briefly, contains the following:

8. An interference between the entangled systems always exists. In the DPM it takes the form of vector interference. (Vector interference is analogous to wave-mechanical interference but is not identical.) Vector interference requires the states of the two systems to be able to be ordered to pairs of the element states, which are called corresponding element states. The entanglement comes out in that the actualizations of the corresponding element states are conditional - the condition of the actualization of the state s1 of the system X being the actualization of the corresponding state s1' of the system $\mathrm{Y}$ and vice versa. Thus, the interference changes the vector $\mathbf{s} 1$ in the system $X$ and the vector $\mathbf{s} 1$ ' in the system $Y$ into the vector which consists of the products of their paired elements, for example:

\section{Element states}

$\begin{array}{rllllll} & \mathrm{s} 1 & \mathrm{~s} 2 & \mathrm{~s} 3 & \mathrm{~s} 4 & \mathrm{~s} 5 & \Sigma \\ \text { s1: } & (.1 & .3 & .2 & .1 & .3) & 1 \\ & \mathrm{~s} 1{ }^{\prime} & \mathrm{s} 2 & \mathrm{~s} 3 & \mathrm{~s} 4 & \mathrm{~s} 5 & \\ \text { Products of pairs: } & (.4 & .1 & .1 & .3 & .1) & 1 \\ \text { Normalized: } & .04 & .03 & .02 & .03 & .03 & .15 \\ & (.27 & .20 & .13 & .20 & .20) & 1\end{array}$


Note the following cases, particularly:

a) If a homogeneous vector interferes with another vector it becomes a copy of that vector.

b) If a vector interferes with a unit vector it becomes a copy of that unit vector. (A unit vector is a vector in which one element is 1 and the others are 0 .)

c) If some vector in the transition probability matrix is such a unit vector, where the diagonal element $\left(\mathrm{p}_{\mathrm{i}, \mathrm{i}}\right)$ is 1 , the evolution process stops in the state $i$. Thus, the state $i$ is a stable one. This stability makes possible the observation of the system, in principle. Therefore we are able to call the state the matter state. (Pure superposition states are not observable.) We call this unit vector the stabilizing vector.

d) If a vector $\mathbf{s}_{\mathbf{i}}$ of the system $\mathrm{S}$ interferes with the stabilizing vector $\mathbf{s}_{\mathbf{i}}$ ' of another system $\mathrm{S}^{\prime}$ and the states $i$ and $i$ ' are corresponding element states, then the vector $\mathbf{s}_{\mathbf{i}}$ becomes a stabilizing vector and the system $\mathrm{S}$ ends in the state $i$ (i.e., the process stops at $i$ ). The system $\mathrm{S}^{\prime}$ functions as a detector and the vector $\mathbf{s}_{\mathbf{i}}{ }^{6}$ is called a detector vector.

This stabilizing process in quantum evolution seems identical with the "collapse of the wave function" in wave mechanics, whereas it is, according to Germine, the same as decoherence (Germine, 2011): "Decoherence is really the same thing as [the] collapse of the wave function." (p. 8).

9. When all element states are included in the corresponding element pairs, as above, a total vector interference is in question. It is, however, logical and natural to generalize the vector interference concept in such a way that it also concerns the systems which are partially entangled (interdependent). This is the situation when, among the element states of two systems, some are corresponding element pairs, while some are not. The interference then occurs as partial vector interference. It is determined by counting the products of the paired elements and then by normalizing the changed vectors. The result is two vectors, for example:

Element states (the corresponding element pairs in boldface)

\begin{tabular}{|c|c|c|c|c|c|c|c|c|}
\hline & s1 & s2 & s3 & s4 & s5 & & & $\Sigma$ \\
\hline \multirow[t]{2}{*}{ s1: } & $(.1$ & .4 & .2 & .1 & $.2)$ & & & 1 \\
\hline & s1' & s2' & s3' & & & s6' & s7 & \\
\hline s1': & $(.2$ & .3 & .1 & & & .3 & $.1)$ & 1 \\
\hline \multirow[t]{2}{*}{ Products of pairs: } & .02 & .12 & .02 & & & & & \\
\hline & & & & & & & & $\Sigma \mathbf{s} 1=.46, \Sigma \mathbf{s} 1 \mathbf{1}^{\prime}=.56$ \\
\hline Normalized: & & & & & & & & $\Sigma$ \\
\hline $\mathbf{s 1} 1_{\text {int }}$ & $(.04$ & .26 & .04 & .22 & $.43)$ & & & 1 \\
\hline s1' ${ }_{\text {int }}$ & $(.03$ & .21 & .04 & & & .54 & $.18)$ & 1 \\
\hline
\end{tabular}

The partial vector interference is a novelty, presented only in the DPM, but rather well justified (see Rainio 2008, pp. 71-73). It plays a central role in the solution of the mind/brain problem - as we shall see later in this article.

\subsection{Theory of Interaction between Consciousness and the Brain}

The key phenomenon from the point of view of the mind-body problem is the brain process called exocytosis. According to Eccles, "exocytosis is the opening of a channel in the PVG (presynaptic vesicular grid) and discharge of the vesicle's transmitter molecules into the synaptic cleft" (Eccles, 1994, p. 154). Thus, exocytosis is an essential condition of the nerve impulse going over the synaptic cleft. Further, Eccles emphasizes that “... the complex process of exocytosis and its probabilistic nature are governed by a trigger mechanism which may involve quantum transitions between metastable molecular states" (p. 154).

Eccles summarizes his hypothesis as follows: "It is not proposed that the mental events initiate activity at a synapse by an excitatory action either on the presynaptic or postsynaptic elements of a synapse... On the contrary, the hypothesis is that the mental events merely alter the probability of a vesicular emission that is triggered by a presynaptic impulse" (p. 73, my italics)

Eccles writes in more detail about the emission: "The units are the synaptic boutons that, when excited by an all-or-nothing nerve impulse, deliver the total contents of a single synaptic vesicle, not regularly, but probabilistically. This quantal emission of synaptic transmitter molecules (about 5000 to 10000) is the ultimate functional unit of the transmission process from one neuron to another" (p. 55). According to Eccles, "the statistical technique... enables the determination of the probability of release by a nerve impulse of a single 
synaptic vesicle. As in simpler situations this probability of release is always less than one, and is in fact very low for the hippocampus with average mean values of $0.27,0.24$, and 0.16 for three completely reliable experiments on the hippocampus. ... This is a fundamentally important finding..." (p. 134). The basic theoretical idea of Eccles is that a mental state influences the probability of exocytosis, increasing it.

\subsection{A Mathematical Model for the Mind/Brain Theory by Eccles}

Our mathematical presentation of Eccles' theory follows the DPM described above (Section 1). That model is one form of DQM (see, for example, Rainio, 2008, and Rainio \& Malaska, 2011).

According to Eccles, the state of a mental system may change, or increase, the probability of exocytosis in one or several synapses. This means that the quantum evolution process (at one synapse) is controlled by a certain transition probability vector, for example:

\begin{tabular}{lll} 
& \multicolumn{2}{c}{$\mathrm{E}$} \\
$\neg \mathbf{E x}$ & $\lceil .8$ & $.2\rceil$ \\
$\mathbf{E x}$ & $\lfloor 0$ & $1\rfloor$
\end{tabular}

(Notation: $\neg$ Ex means: "no exocytosis", Ex means: "exocytosis occurs".)

The basic mathematical hypothesis is that the change of the probabilities occurs as a consequence of a vector interference between two vectors: the probability vector of exocytosis $(\neg \mathbf{E x})$ and the mental state vector $\mathbf{m}$. The interference will, in this context, be a partial vector interference. To activate the brain to a certain action (for example, to a motor performance), a large number of synapses, however, need to go through exocytosis. This bundle of synapses is called a dendron. Eccles argues that the simultaneous exocytosis of all synapses in a dendron is produced by a corresponding bundle of mental states. He calls that bundle a psychon.

Note: just this hypothesis and the concept of a "psycho" have aroused criticism of Eccles' theory. We shall see whether the term is useful or not.

The DPM explains the rise of probabilities in the whole dendron in an understandable way. It is assumed that one, and only one, mental state vector is in partial vector interference with several synaptic exocytosis vectors. These synapses are included in one dendron or in several of them; no special psychon concept is needed in this context. The following example illustrates the interference effect of a mental state on several synapses simultaneously. (There are only three synapses in the example. If one would like to make the case a little more 'realistic', one would have to take millions or billions of synapses into account. Using only three of them may, however, show the idea.)

Let $\mathbf{m} 1$ of system $\mathrm{M}$ be a state vector of a mental state $\mathrm{m} 1$ :

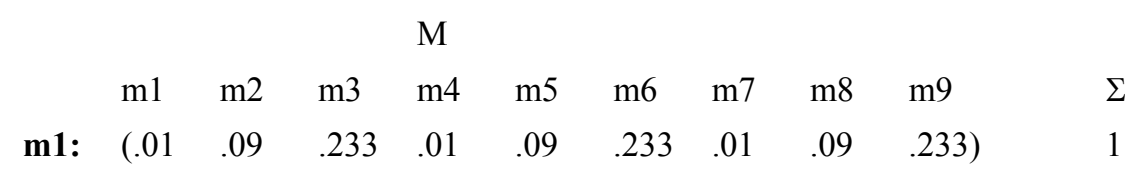

Let $\neg \mathbf{E x}$ be the exocytosis vectors of the 3 synapses S1, S2, and S3:

\section{S1}

$\neg$ Ex Ex

$\neg \mathbf{E x}:\left(\begin{array}{ll}.8 & .2\end{array}\right)$
S2

$\neg$ Ex $\quad$ Ex

$\left(\begin{array}{ll}.8 & .2\end{array}\right)$
S3

$\neg \mathrm{Ex} \quad \mathrm{Ex}$

$\left(\begin{array}{ll}.8 & .2\end{array}\right)$

Notice that the vector $\neg \mathbf{E x}$ of synapse S1 is ordered above to correspond to the states $\mathrm{m} 1$ and $\mathrm{m} 2$ of the vector $\mathbf{m} 1$, the vector of synapse $\mathrm{S} 2$ to the states $\mathrm{m} 4$ and $\mathrm{m} 5$, and the vector of synapse $\mathrm{S} 3$ to the states $\mathrm{m} 7$ and $\mathrm{m} 8$. Thus, the states $\mathrm{m} 3, \mathrm{~m} 6$, and $\mathrm{m} 9$ do not correspond to any states.

Let us compute the (simultaneous) partial vector interference of the vector $\mathbf{m} \mathbf{1}$ and the vectors $\neg \mathbf{E x}$ of the synapses $\mathrm{S} 1, \mathrm{~S} 2$, and $\mathrm{S} 3$ :

Interference between the mental state vector and 3 exocytosis vector:

$\begin{array}{lllllllllll}\mathbf{m 1}: & (.01 & .09 & .233 & .01 & .09 & .233 & .01 & .09 & .233) & \Sigma=1 \\ \neg \mathbf{E x}: & (.8 & .2) & & (.8 & .2) & & (.8 & .2) & & \end{array}$




\begin{tabular}{rlllllllll} 
Pair products: .008 & .018 & .233 & .008 & .018 & .233 & .008 & .018 & .233 & $\Sigma=.778$ \\
$\mathrm{~S} 1$ & & & $\mathrm{~S} 2$ & \multicolumn{7}{c}{$\mathrm{S} 3$} \\
.008 & .018 & & .008 & .018 & & .008 & .018 & & \\
$\Sigma \mathrm{S} 1=.026$, & & $\Sigma \mathrm{S} 2=.026$, & \multicolumn{2}{c}{$\Sigma \mathrm{S} 3=.026$} &
\end{tabular}

Normalized exocytosis vectors:

$\neg \mathbf{E x} \mathbf{x}^{\prime}: \quad\left(\begin{array}{llll}.31 & .69\end{array}\right) \quad\left(\begin{array}{lll}.31 & .69\end{array}\right) \quad(.31 \quad .69)$

Normalized m1'-vector:

m': $\quad\left(\begin{array}{lllllllll}.01 & .023 & .30 & .01 & .023 & .30 & .01 & .023 & .30\end{array}\right) \quad \Sigma=1$

The normalized $\neg \mathbf{E x}$ ' vectors show that the exocytosis probabilities have increased from .2 to .69. Thus, applying the idea of vector interference between two systems (mental and physical, the trigger system), we have succeeded in giving a mathematical explanation of exocytosis - an explanation that is more exact than Eccles' verbal description.

\subsection{Binding Problem}

The psychon idea of Eccles is seemingly vague and has provoked a lot of critics, as already stated. Perhaps the mental state vector could be called a psychon - it actually corresponds well to the description of a psychon by Eccles. Notice, however, that in DPM presentation there is no need to assume any one-to-one correspondence between psychon elements and the synapsis elements of a dendron.

It is reasonable to assume that in the mental state vector there are as large a number of state elements as are needed for a simultaneous interference connection, even to all the systems in the brain. One has to notice that this hypothesis gives a solution to the so-called binding problem, which can be described in the following way: "How the unity of conscious perception is brought about the distributed activities of the central nervous system" (Revonsuo \& Newman, 1999, p. 123).

It is exactly the binding problem which has caused troubles to brain researchers. The answer given in terms of DPM is simple: the solution cannot be found in material brain mechanisms but in the mental state vector. The problem is not one of the traditional (classical) physics but quantum mechanical - and solvable, provided that the idea of partial vector interference is acceptable.

In Section 2, one direction of the interaction between the mind and the brain is treated, namely how mental states affect matter (brain cells). The other side - how the matter (stimuli of the sense organs and, consequently, the flow of impulses in synapses) affects mental states - i.e., perception - now needs to be analyzed. This will be done in Section 3.

\section{The Perception Process in Terms of DQM}

In this section the two main functions of perception - attention and coding - are examined according to Eccles' description. There is now the possibility of putting the basic problem of the perception process in such a conceptual form that an application of the DPM can be used. With the mathematical model an opportunity then opens up to go further in the analysis of cognitive processes, first to examine gestalt generation and then to treat one particular case of it, reversible figures. The analysis of these looks like an insignificant detail of cognition but in this context it is important. In this kind of research it is an exceptional case which gives an opportunity to produce estimates of empirical data.

\subsection{The Course of the Perception Process. Attention}

The evolution of perceptions from impulses requires, at the lowest level, two things:

1) the activation of certain receiving brain areas (the increase of the exocytosis probabilities in the brain areas in question), i.e., attention and

2) the accumulation of information concerning the number of impulses going over the synaptic clefts, as well as their relative frequencies (coding to the mental system).

Both of these can occur only through interferences.

\section{Attention:}

According to Eccles, attention means the activation of large brain areas by the subject. He writes: “... attention causes neural activity in rather large areas of the brain, as is revealed by the rCBF technique (regional cerebral blood flow) (Roland 1981).” (p. 99); “... mental act of attention can activate appropriate regions of the cerebral 
cortex.” (p. 100); “... a self is able by attention to activate any selected parts of the neocortex at will.” (p. 174); “... our attention by psychon activation will enhance the range and intensity of the dendron activation for increasing the visual input and discrimination. It can be recognized that by attention we enhance and embellish our perception." (p. 175).

In the frame of reference of the DPM the fact that attention causes neural activity in large areas of the brain means that the subject produces autonomously ("at will") a mental system which interferes with the synapse vectors, even in the area of the whole brain.

How does attention go further? If stimulus impulses come out in some area of the brain when the attention is aroused, the increase in mental activation (Eccles: "psychon activation") heightens the probabilities of exocytosis in these areas (Eccles: "... will enhance the range and intensity of the dendron activation"). This makes the impulses "flow" more densely in these synapses.

During the perception process, attention is the first phenomenon in which the mental world "comes towards" physical occurrences. The second will be a registering or coding system of impulses; those systems will collect information on the density of the impulses coming from the sense organs, of the variation in them, and of the structure of their flow. All this comprises the "hard problem of perception"; Eccles has somehow neglected it.

\subsection{The Basic Problem of the Perception Process}

The interaction between the mental systems always occurs through vector interferences, as well as the mental system M and exocytosis system (or "trigger system"), which was already described in Section 2.3. Eccles' analysis is focused on motor activity while the sensory processes receive less interest in his presentation. He does not describe in detail the interaction between sensory dendrons and mental systems in which the coding of sensory impulses happens.

No fundamental problems exist regarding the application of Eccles' theory to the motor behaviour when the DPM is used: both the state vector of the mental system and the state vector of the trigger system are described as superpositions and, thus, could interfere. But what about the perception process?

Our starting point is a classic macro-physical event: the increase in the number of neuronal impulses which is produced by the sense organ. How is this material (chemical) event in the brain able to produce an effect on the consciousness, on the mental state of the subject? How can a change in a material impulse system cause a change in a mental system? Notice that the $\neg \mathbf{E x}$ vector of the trigger system does not change as a consequence of the number of vesicles in the presynaptic dendrite ("bouton") increasing, not at all. The trigger system (exocytosis system) is a quantum system and such a system can only be changed by an interference.

On page 89 of his book Eccles promises to present a solution to the basic problem of perception: “... there will be developed a theory of perception from dendron activity to psychon experience, also on the basis of quantum mechanics." This promise is fulfilled only superficially, however, on two pages in Section 6.9., "How Neuronal Activity in the Sensory Systems Could Evoke Conscious Perceptions" (pp. 108-109). He writes: "The response of the neocortex to attention is preparatory to the transaction whereby dendrons are activated in the perceptual process to produce the perceptual mental events. For example, it can be asked: how can activated dendrons of the tactual system give rise to some specific tactual perception?" (p. 108).

Eccles thus seems to think that the dendrons, activated by the sensory process, would somehow immediately "produce" (or "give rise" to) perceptual mental events. How could something material (the impulses) produce something mental, straight away? Unlike in his description of the generation of motor processes, Eccles is not able to give a clear detailed presentation here.

In this same context Eccles writes: “... [the] psychon is presented with an increase in its dendron of vesicles available for exocytosis in accord with selection by means of the quantal probability field. The hypothesis is that each such exocytosis is a 'success' for the psychon, which gives a signal that is transmitted into the mental world." (p. 108). In which way does the psychon actually get the information of "an increase in its dendron of vesicles available for exocytosis"? Seemingly, it is more a question of the number of vesicles going over the synaptic cleft than the number of vesicles in a dendron ("available for exocytosis").

A key word in Eccles' text seems to be "success", but what does this actually mean? The same indistinctness turns up in Eccles' presentation through the whole section. "In the reverse transaction (perception), brain to mind, it is necessary to have an extension of the hypothesis, namely that every time a psychon successively selects a vesicle for exocytosis (in accord with the quantal probability field) the 'micro-success' is registered in the psychon for transmission through the mental world." (p. 109). 
What is meant by "micro-success" and what "registration" is remain open questions, but it seems to be important to notice the emphasis on "success" in Eccles" text. One may guess that Eccles has here noted the need for an extra hypothesis. He does not present that hypothesis, however.

\subsection{A Proposal for a Solution of the Basic Problem. A Mathematical Model for the Perception Process}

In the previous analysis we reached the conclusion that the basic problem of perception is to transfer the information about the impulses crossing over the synapses for the use of the mental systems In addition to that, one should notice that only interferences can change the state vectors.

Eccles gives the following "program list" about the flow of the perceptional process (concerning the tactual perceptions in this case):

1. Background activation by attention to the tactual area.

2. Sensory input into the tactual nervous system.

3. Activation in the neocortex of the dendrons of the tactual system.

4. Increased exocytosis from the presynaptic vesicular grids of the pyramidal cells of these dendrons. This gives increased opportunities for selective exocytosis by the linked psychon, which is in accord with a quantum probability field.

5. The increase in vesicular selection by the psychon for touch gives directly the experience of a tactual perception... and a psychon 'success' signal for transmission and integration...

All other perceptions of the outer sense can be similarly explained by appropriate attention.” ( p. 108)

This "Eccles program" can be presented, mutatis mutandis, in the form of a DPM description in the following way:

1. Backgroud activation by attention means an interference between a particular attention system (system A) and the trigger systems (Ex) in large areas of the brain. This causes an increase in the probability of exocytosis in all synapses concerned. For example (in the case of one synapse):

a) Ex-system in neutral state:

$$
\begin{array}{rlr} 
& \text { Ex } & \\
& \neg \mathrm{Ex} & \text { Ex } \\
\neg \mathbf{E x} & .8 & .2
\end{array}
$$

b) Attention system:

$$
\begin{array}{rll} 
& \text { Att } & \\
& \neg \mathrm{Ex} & \text { Ex } \\
\neg \mathbf{E x} & .4 & .6
\end{array}
$$

Computing the interference:

$\begin{array}{llll}\text { Pair products: } & .32 & .12 & \Sigma=.44 \\ \text { Normalized: } & .73 & .27 & \Sigma=1\end{array}$

Attention, i.e., the interference with the A-system, has thus increased the probability of exocytosis (in this synapse) from .2 to .27 . This happens in every synapse in the area of the brain activated by the attention.

2 and 3. "Sensory input" means the increase in the number of sensory impulses in the synapses, particularly the increase in the frequency of impulses coming to the bouton, the presynaptic part of the synapse, at a certain time interval.

4 and 5. Eccles seems to think that the increased number of exocytoses as such directly causes the increase in the corresponding probabilities. There are no grounds for that. One has to build a special model which produces that result.

We have available for our use the concept of the stimulus threshold (or perception threshold). There already exists in our sense organs an "all-or-nothing" phenomenon: if the intensity of a stimulus remains below a certain threshold, no perception occurs. It can be put in a more detailed manner: if (in an afferent neuron) the number of impulses going over the synaptic clefts (during a certain time interval) is higher than the perception threshold, this chain of impulses produces in a sensory center a state which is called (following the idea of Eccles) a success state, Succ $_{\mathrm{syl}}$. Correspondingly, if the threshold is not crossed, the state will be a non-success state, $\neg$ Succ $_{\text {syl }}$. One can give a particular meaning to the term "success": reward in trial-and-error learning. This way of using the term seems to be in accordance with Eccles' view.

The hypothesis of learning is rather speculative in this "thought experiment" but is not without grounds. (It is actually obvious that this kind of feedback occurs, not only in the afferent neuronal system but also in motor functions, although Eccles does not take it into account in his work.) 
In detail the hypothesis is as follows: If a synapse sy1 has caused the Succ-state, then a certain "learning vector" $\Lambda_{\text {syl }}$ interferes with the vector $\neg \mathbf{E x}$. If sy1 has caused the $\neg$ Succ-state, there will be no change in the $\neg \mathbf{E x}$ vector, i.e., it interferes but with a homogeneous vector. In vector $\Lambda_{\mathrm{sy} 1}$, particularly, $\mathrm{p}_{\mathrm{Ex}, \mathrm{Ex}}<\mathrm{p}_{\mathrm{Ex}, \neg \mathrm{Ex}}$, i.e., the interference increases the probability $\mathrm{p}_{\mathrm{Ex}, \neg \mathrm{Ex}}$.

\begin{tabular}{|c|c|c|c|c|c|c|}
\hline \multirow{3}{*}{ State: } & \multicolumn{2}{|l|}{$\mathrm{Ex}_{\mathrm{sy} 1}$} & \multicolumn{2}{|l|}{$\mathrm{Ex}_{\mathrm{sy} 2}$} & \multicolumn{2}{|l|}{$\mathrm{Ex}_{\mathrm{sy} 3}$} \\
\hline & Success & & Succes & & $\neg \mathrm{Suc}$ & cess \\
\hline & $\neg \mathrm{Ex}$ & Ex & $\neg \mathrm{Ex}$ & Ex & $\neg \mathrm{Ex}$ & Ex \\
\hline$\neg \mathbf{E x}$ & .73 & .27 & .73 & .27 & .73 & .27 \\
\hline$\Lambda$ & .4 & .6 & .4 & .6 & .5 & .5 \\
\hline
\end{tabular}

Interference:

Pair products: $\quad .29 \quad .16 \quad \Sigma=.45 .29 \quad .16 \quad \Sigma=.45 \quad .365 \quad .135 \Sigma=5$

$\begin{array}{lllllll}\text { Normalized: } & .64 & .36 & .64 & .36 & .73 & .27\end{array}$

As a result of the rewarding learning, the exocytosis probability has increased to .36 in synapses 1 and 2 but retained the value of .27 in synapse 3 .

The successive changes in the probabilities continue as long as impulse chains exist that are intensive enough to go over the threshold. For example, after six rewarding learning events the vector $\neg \mathbf{E x}$ would get the form: $(.20, .80)$.

Because the information of all synapses which belong to the attention area needs to be integrated, it will be assumed that at the lowest level of perception a homogeneous vector $\mathbf{m}_{\mathbf{I}} \mathbf{1}$ exists. This vector is in partial vector interference with all the synapse vectors that have been mentioned (i.e., $\neg \mathbf{E x}$ vectors). The vector is called a diffuse perception contents vector or profile - diffuse because it is not differentiated in any gestalt generation process. An example of its computation of in the case of five synapses is presented below:

Computing the diffuse perception contents vector:

Synapses sy 1 - sy 3 are the objects of attention and rewarding learning, while sy4 and sy5 are objects of attention only.

$\begin{array}{llllllllllll} & \text { sy1 } & & \text { sy2 } & & \text { sy3 } & & \text { sy4 } & & \text { sy5 } & & \\ \neg \mathbf{E x} & (.2 & .8) & (.2 & .8) & (.2 & .8) & (.73 & .27) & (.73 & .27) & \\ \mathbf{m}_{\mathbf{I}} \mathbf{1} & (.1 & .1 & .1 & .1 & .1 & .1 & .1 & .1 & .1 & .1) & \Sigma=1\end{array}$

Interference:

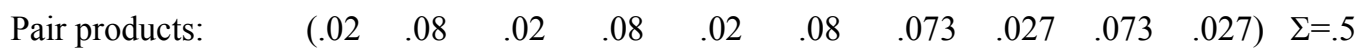

Normalized

$\mathrm{m}_{\mathbf{1}} \mathbf{1}^{\prime}$ :

$\left(\begin{array}{llllllllll}.04 & .16 & .04 & .16 & .04 & .16 & .146 & .054 & .146 & .054) \\ \Sigma & =1\end{array}\right.$

The vector $\mathbf{m}_{\mathbf{I}} \mathbf{1}$ is a state vector of the system $\mathrm{M}_{\mathrm{I}}{ }^{\prime}$ at (the lowest) mental level. The transition probability matrix of this system consists, in addition, of all other state vectors of this system at that level; they are all homogeneous.

It may well be assumed that all other state vectors $\mathbf{m}_{\mathbf{I}}$ interfere with the diffuse perception contents vector, $\mathbf{m}_{\mathbf{I}} \mathbf{1}$ ' The transition probability matrix of the system M1 will then have the following form:

\begin{tabular}{|c|c|c|c|c|c|c|c|c|c|c|}
\hline & & & & & $\mathbf{M}_{\mathbf{I}}{ }^{\prime}$ & & & & & \\
\hline & $\mathrm{m} 1$ & $\mathrm{~m} 2{ }^{\prime}$ & m3' & $\mathrm{m} 4$ & m5, & m6' & $\mathrm{m} 7$ & $\mathrm{~m} 8{ }^{\prime}$ & m9' & m10' \\
\hline $\mathrm{m}_{\mathbf{I}} \mathbf{1}^{\prime}:$ & $\lceil .04$ & .16 & .04 & .16 & .04 & .16 & .146 & .054 & .146 & $.054\rceil$ \\
\hline m $\mathbf{m}^{\prime}$ : & & same & row as & above & & & & & & \\
\hline m m $^{\prime}:$ & & & -"- & & & & & & & \\
\hline .. & $\ldots$ & $\cdots$ & & & & & & & & \\
\hline $\mathrm{n}_{\mathbf{I}} 10^{\prime}:$ & L.04 & .16 & .04 & .16 & .04 & .16 & .146 & .054 & .146 & ل.054. \\
\hline
\end{tabular}

The matrix $\mathbf{M}_{\mathbf{I}}$ ' is the eigenmatrix of the system $\mathbf{M}_{\mathrm{I}}$, because all the row vectors (state vectors) are identical. 
Note: There is another way to arrive at the use of eigenmatrix. If a long enough time-step is chosen the matrix takes approximately the form of the eigenmatrix.

Now it is important to notice: When the evolution process of a quantum system occurs, it proceeds, or "jumps", from one superposition to another, but all states are now identical; the system's state does not actually change during the process; in other words, the state is always the superposition given by the row vector with the same probability elements.

The result is that the system remains in that mental state the diffuse perception contents vector describes. This state is not "definite" in a quantum mechanical sense (because it is a superposition). It is called the invariant process state of the perception contents or, in brief, the invariant process state.

There are many quantum mind presentations (e.g., by David Bohm) where different "levels" of the mental world are mentioned but not described in detail. In DPM, a basic assumption is that an invariant process state at a lower level may exist in a state at a higher level as its intrinsic property.

As an analogous example of the relationship of quantum mechanical systems at two different levels let us look at electron and atom systems: at the level of elementary particles, each electron system has its own evolutionary process, which is determined by the transition probabilities in the eigenmatrix. In their stationary evolution process the electron systems, when transiting from one state to another, actually repeat the same superposition state - analogously to what is presented above. Although the processes in the electron systems proceed in their own way, the system at the higher level, the atom system, stays constantly in one superposition state. There exists an intrinsic property of the atom system that the electrons stay in a certain stationary process.

The state vector at the higher level (II) is presented in the following way:

$$
\begin{array}{llllcc} 
& & \multicolumn{5}{c}{\mathbf{M}_{\text {II }}} & \\
& \mathbf{m}_{\text {II,1 }} & \mathbf{m}_{\text {II, }, 2} & \mathbf{m}_{\text {II,3 }} & \mathbf{m}_{\text {II,4 }} & \ldots \mathbf{m}_{\text {II,n }} \\
& (\approx 1 & \approx 0 & \approx 0 & \approx 0 & \ldots \approx 0)
\end{array}
$$

where $\mathbf{m}_{\mathrm{II}, 1}$ is that state which appears at the first level as the evolution process of the perception contents system $\mathrm{M}_{\mathrm{I}}{ }^{\prime}$. The probability $\approx 1$ means a relatively stable state. It is reasonable to think that it remains for as long a time as there is no essential change in the contents of what is perceived.

It is tempting to make the radical assumption that the intrinsic properties at that second level would be experienced. (This way of thinking is according to Chalmers' view. This will be discussed later in this article, in the Discussion section.) An argument for that is that the relative stability of the state gives an opportunity for that. In addition, the perceived process is invariant.

Thus, the mental system at the second level can be called the experience system at the lowest level, as well as the diffuse perception system. "Diffuse" here means that the perception contents appear as an undifferentiated fusion of parts which has no interpretation as a gestalt. The real interpreting gestalt process does not begin until the subject in an (autonomous) seeking process finds at the third level a system which has a state that corresponds to the state $\mathbf{m}_{\mathbf{I I}, \mathbf{1}}$ and some other state that differentiates it. Thus, the process produces an experience of a well-differentiated gestalt (as an intrinsic property of the state).

The transition probability matrix of the system at the third level could be, for example:

$\begin{array}{lll} & & \mathbf{M}_{\text {III }} \\ & \mathrm{m}_{\text {III, } 1} & \mathrm{~m}_{\text {III,2 }} \\ \mathbf{m}_{\text {III, } 1} & \left\lceil\mathrm{p}_{1,1}\right. & \mathrm{p}_{1,2} \\ \mathbf{m}_{\text {III,2 }} & \lfloor\approx 0 & \approx 1\rfloor\end{array}$

It is reasonable to assume the probability $p_{1,1}$ relatively small, because the gestalting process (transition to state 2 ) is supposed to be a rather rapid event. The undifferentiated perception contents at the lower level, the second level, now appear as the state $\mathrm{m}_{\mathrm{III}, 1}$ at the third level but, according to psychological findings, these perceptual contents will be generated as "some gestalt" very quickly. The gestalting process transfers the system to a new state, to state $\mathrm{m}_{\mathrm{III}, 2}$ in this case. The probability $\approx 1$ indicates that this state remains relatively stable. This means, according to the DPM, that there will be, in experience, enough time for the "subject" to "examine" the intrinsic properties of the state and, thus, to differentiate and interpret it further.

\subsection{Gestalt Generation}

\subsubsection{Mental Systems Own World}

Gestalt psychology, particularly, has emphasized the activity of the subject in the perception process. The subject determines what will be perceived and he is autonomic in that. The stimulus impulses form only certain limiting conditions. 
Note: An exact definition of the subject is not possible at this stage of research. We make only that superficial assumption that there exists (at a very high level) some (autonomous) system on which the participation of the mental systems in the consciousness process depends. This kind of system is the fundamental decision-making system ("Life Space"; see, e.g., Rainio, 2008, pp. 79-80, and Rainio, 2009b). We do not have, however, any detailed answer to the theoretical question about the existence of the subject.

The projection of processes from one level to another and the interference chain described above form, in principle, the DPM model for perception, for gestalt generation, and for the interpretation of the perception.

Self-perception (introspection) tells us that the formation of the gestalt and giving meaning to the perceived object (interpretation) belong closely together: at some stage of the formation of the gestalt the experience also includes comprehending the meaning of the gestalt, some interpretation, but not necessarily verbal. The meaning thus belongs among the intrinsic properties of the system.

The mental systems at the first level seem to correspond to the "psychons" of Eccles, the difference between his description and the DPM lying in the fact that Eccles assumed the psychons to be in a one-to-one relationship to the dendrons. (Eccles seems to give up this assumption later in his text, as we shall see.)

Eccles' "own world of the psychons": it is interesting to notice that in some contexts Eccles clearly points to an "own world of the psychons", independent of the dendrons, when he describes the "psychon world": “... some psychons may be linked only with psychons.” (p. 109); “... psychons may exist apart from dendrons in a unique psychon world of the self...” (p. 179); “... a fundamental question: are the experiences of the self also composed of unitary psychons in the same manner as for perceptual and other experiences? If so, is each of these psychons also linked with its dendron, and where in the neocortex are these dendrons? We can further ask if there is a category of organized psychons not linked to dendrons, but only with other psychons forming a psychon world apart from the brain." (p. 179).

\subsubsection{Gestalts as Organized Perceptions}

It is a general finding in developmental psychology that perception does not come out as a ready picture of reality but it is a process which proceeds from a diffuse gestalt to a differentiated one (so-called "actual genesis"). The result is never completely differentiated. It is dependent on the individual's developmental level and the situational possibilities.

Differentiation according to a scheme:

What is seen in the following figures?

A
$\mathrm{B}$

\section{$\mathrm{C}$}

Presumably, one sees in A a cheerful face and in B a sour face. The immediate gestalt formation occurs according to this kind of scheme. Only afterwards may one analyze the forms and, after deep reasoning, come to the result that the "faces" are actually drawings made of very simple lines.

In A and B some kind of glance, a stare into the reader's eyes, is perceived. But the same is true in so simple a case as $\mathrm{C}$, too. This is understandable for psychological reasons: the perception of the staring eyes of an enemy has been always extremely important and learned quickly. What about a modern person? We may say that "he has beneath his culture the ability to see in the manner of a primitive man". In instinctive behaviour, gestalt formation according to schemes is a central feature.

It seems to be necessary to presume that elementary scheme-based perception already occurs at the second mental level - so that the process of system $\mathrm{M}_{\mathrm{II}}$ transits from a diffuse state to a differentiated state corresponding to the scheme. One has to write the matrix $\mathbf{M}_{\mathbf{I I}}$ in a new form, for example as follows:

\begin{tabular}{|c|c|c|c|c|c|}
\hline & & $\mathbf{M}_{\mathrm{II}}$ & & & \\
\hline & $\mathrm{m}_{\mathrm{II}, 1}$ & $\mathrm{~m}_{\mathrm{II}, 2}$ & $\mathrm{~m}_{\mathrm{II}, 3}$ & $\mathrm{~m}_{\mathrm{II}, 4}$ & $\ldots \mathrm{m}_{\mathrm{II}, \mathrm{n}}$ \\
\hline $\mathbf{m}_{\mathrm{II}, 1}$ & $\lceil .05$ & .95 & $\approx 0$ & $\approx 0$ & $\ldots \approx 0$ \\
\hline $\mathbf{m}_{\mathrm{II}, 2}$ & .05 & .95 & $\approx 0$ & $\approx 0$ & $\ldots \approx 0$ \\
\hline & L.05 & .95 & $\approx 0$ & $\approx 0$ & $\ldots \approx 0$ \\
\hline
\end{tabular}

The matrix is meant to show that the invariant process state as such (the state $\mathrm{m}_{\mathrm{II}, 1}$ ) - as a completely diffuse "stuff" - does not stay long but transits to another, more stable state, $\mathrm{m}_{\mathrm{II}, 2}$, where the perception contents are 
differentiated according to the scheme. This means that the subject now experiences the staring glance (without any verbal interpretation). Self-perception tells the subject that it is reasonable to assume the state $m_{I I, 1}-$ the experience of totally undifferentiated perception contents - to be very brief, because the subject does not manage to notice it. On the contrary, the state of experiencing the "staring", the state $\mathrm{m}_{\mathrm{II}, 2}$, is something rather stable, so that the subject manages to create a self-perception of it, to experience the "staring".

The matrix $\mathbf{M}_{\mathrm{II}}$ is an eigenmatrix and consequently in the evolution process of the quantum mechanical system $\mathrm{M}_{\mathrm{II}}$ the same superposition state is repeated. It is again an invariant process state and, analogously with the earlier case, the transfer from the first level to the second level, the evolution process of the system $\mathrm{M}_{\mathrm{II}}$ at the second level appears as an intrinsic property of the state $\mathrm{m}_{\mathrm{III}, 1}$ at the third level. This state is, thus, an element state in the transition probability matrix of the system $\mathrm{M}_{\mathrm{III}}$.

It is logical to think that, at this higher level, there appears the experience of meaning and possibly a verbal interpretation linked to that. Thus, in our example, the undefined and undifferentiated impression of being an object of a kind of "staring" would be interpreted at this higher level as "a figure which expresses a staring face".

When does the perception process end? Obviously, at the moment when the quantum mechanical invariant process ceases. Then the states at the higher levels also lose their experiential features. The perception process at the lowest level stops, of course, when the flow of the stimulus impulses ends (when the subject closes his eyes or shuts his ears or when the subject fixes his attention in a new direction). There are, however, phenomena which require further theorizing, for example: afterimages, eidetic facts, and hallucinations.

So far only some basic principles of the generation of perception have been analyzed. We could go further and try to describe human thinking, imagination, fantasy, decision making etc. but at this stage of analysis that would lead us deeper and deeper into speculation and out beyond the range of this article. Instead of that, an examination will be performed of how the DQM model may fit the empirical facts and one simple example of that will be shown in the following section.

\subsubsection{An Application of the DPM: Perception of Reversible Figures}

\section{About the reversible figures:}

Look at the so-called Rubin's figure, presented in Fig. 3. What do you see? Is there a) a white vase against a black background or b) two human profiles against a white background? One notices very soon that it is possible to jump from seeing the picture in way $a$ to seeing it in way $b$ and vice versa but they cannot be seen simultaneously in ways $a$ and $b$.

Notice, particularly, that when the figure reverses, no change happens in the physical world; the drawing does not change. The stimulus input has remained exactly the same, while the perception is totally different. The gestalt generation of reversed figures shows irrefutably that in consciousness events are possible which do not have any cause in the material world. Classical physics does not explain this phenomenon. Does quantum mechanics explain it? We will try to find an answer to that.

\section{Gestalt formation in the case of reversible figures:}

How do we understand, according to the DPM, that in the case of the reversible figure, the perception process takes the position either of the vase or the profiles?

In Section 3 the gestalt formation process was presented up to the diffuse stage. There the system $M_{I}$ at the first level was presumed first. Its state $\mathrm{m}_{\mathrm{I}, \mathrm{i}}$ was named the invariant process state. Its projection was a relatively stable state of the system $\mathrm{M}_{\mathrm{II}}$ at the second level. Further, it was assumed that this stability makes it possible to experience its intrinsic properties. At the level concerned, this experience is, however, extremely diffuse (although holistic). In the case of Rubin's figure (Fig. 3) the experience produced by the state $\mathrm{M}_{\mathrm{II}, 1}$ was diffusely "something undefined and black and white" (but without any kind of verbal interpretation).

Our introspection tells us, however, that the totally diffuse gestalt is not long in duration; a very quick transition from this state $\mathrm{m}_{\mathrm{II}, 1}$ occurs. There now exist two equal alternative states: $\mathrm{m}_{\mathrm{II}, 2}$, i.e., the perception of the vase, and $\mathrm{m}_{\mathrm{II}, 3}$, i.e., the perception of the profiles. These are both relatively stable states. Thus, in the case of this reversible figure, we can draw the conclusion that the transition probability matrix of the gestalt generation system has to be written in the following form: 


\begin{tabular}{lllll} 
& & \multicolumn{3}{c}{$\mathbf{M}_{\text {II }}$} \\
& & $\mathrm{m}_{\text {II,1 }}$ & $\mathrm{m}_{\mathrm{II}, 2}$ & $\mathrm{~m}_{\mathrm{II}, 3}$ \\
$\mathrm{~d}:$ & $\mathbf{m}_{\mathrm{II}, 1}$ & $\lceil .02$ & .49 & $.49\rceil$ \\
$\mathrm{v}:$ & $\mathbf{m}_{\mathrm{II}, 2}$ & .02 & .98 & 0 \\
$\mathrm{p}:$ & $\mathbf{m}_{\mathrm{II}, 3}$ & $\mathrm{~L} .02$ & 0 & $.98\rfloor$ \\
& $(\mathrm{v}=$ "vase", $\mathrm{p}=$ "profiles", $\mathrm{d}=$ diffuse $)$
\end{tabular}

The matrix tells us that the perception process transits from the diffuse state with the same probability (.49) to the gestalt formation of the vase as to that of the profiles (.49). In both of them the process remains in a relatively stable state (.98) but can transit back to the diffuse state with a small probability (.02).

It seems realistic to think that the gestalt formation cannot transfer directly from the vase to the profiles or vice versa, i.e., from a state which is a differentiated whole A directly to a state which is a totally other differentiated whole B. (See the zeroes in the matrix.)

Assuming that the time-step has a value in physical time (for example in seconds), it is possible, using this transition model, to compute estimates for the length of the periods which the subject spends in each state.

Using introspection and registering time one can actually create many fruitful experiments with reverse figures and get answers to numerous questions that until now have been left open. One of these is the reverse time, the time the subject remains in a relatively stable state. That will be analyzed in detail below.

The Necker cube. Learning process of reversing the figures (See Fig. 4):

Toppino and Long have examined the reversing speed of a reversible figure using a rotating Necker cube (Toppino \& Long, p. 42, Fig. 2a). They give some experimental results. The frequencies of reverses in periods of $30 \mathrm{sec}$ were the following:

$\begin{array}{lllllllll}\text { Period: } & 1 & 2 & 3 & 4 & 5 & 6 & 7 & 8 \\ \text { Reverses, means: } & 2.5 & 3.5 & 3.8 & 4.2 & 4.3 & 4.4 & 4.6 & 4.7\end{array}$

It is easy to see that the reverse frequencies follow a learning law, specifically Bush's and Mosteller's one operator model (see, e.g., Rainio, 2006, p. 53). The data will be analyzed according to that.

The dynamics of the reverse of the figure are presented, using the DPM, with a transition probability matrix:

$\begin{array}{llll} & & \mathbf{M}_{\text {II }} \\ & \mathrm{d} & \mathrm{a} & \mathrm{b} \\ \text { d } & \lceil .0 & .50 & .50\rceil \\ \text { a } & .16 & .84 & 0 \\ \text { b } & \lfloor .16 & 0 & .84\end{array}$

where $\mathrm{d}=$ diffuse gestalt formation, $\mathrm{a}=$ differentiated gestalt $\mathrm{a}$, and $\mathrm{b}=$ differentiated gestalt $\mathrm{b}$.

The reverse of the figure happens with two time-steps, as a transition on the path a-d-b or b-d-a, depending on whether the starting state of the pair of steps was a or $b$. Thus, the probability of the reverse occurring in a period of two steps is $\mathrm{p}_{\mathrm{rev}}=\mathrm{p}_{\mathrm{a}, \mathrm{d}} \cdot \mathrm{p}_{\mathrm{d}, \mathrm{b}}=\mathrm{p}_{\mathrm{b}, \mathrm{d}} \cdot \mathrm{p}_{\mathrm{d}, \mathrm{a}}$ and in our example: $\mathrm{p}_{\mathrm{rev}}=.16 \cdot .5=.08$.

There are no direct means to determine the length of the time-step in physical time, but one may begin with $1 / 2$ sec and examine what the results would be.

A period of two steps takes $1 \mathrm{sec}$ and there are 30 of those periods in a measurement series. The expectation value of reverses in one period is, thus, $30 \cdot 0.08=2.4$. This fits the empirical data well.

Every event of reversing the figure seems to increase that tendency, i.e., the transition probability from the state a to the state $\mathrm{d}$ (or from $\mathrm{b}$ to $\mathrm{d}$ ) increases following the law of Bush's and Mosteller's operator model of learning: $\mathrm{p}_{\mathrm{t}+1}=\mathrm{p}_{\mathrm{t}}+\alpha\left(\lambda-\mathrm{p}_{\mathrm{t}}\right)$, where $\lambda$ is the learning asymptote $(0 \leq \lambda \leq 1)$ and $\alpha$ the learning coefficient $(0<\alpha<1) ; \alpha$ determines the learning speed.

The learning process is easily simulated and the value of $\alpha$ found by range finding. In every case, the best value of $\alpha$ is .25 . The starting values of the transition probabilities and the value of learning asymptote are dependent on the chosen length of the time-step in the simulation. The learning asymptote is $2 \cdot p_{a, d} \cdot-$ The simulation gives the following results: 
Learning process in figure-reversing:

$$
\begin{aligned}
& \mathrm{p} 1=\mathrm{p}_{\mathrm{a}, \mathrm{d}}=\mathrm{p}_{\mathrm{b}, \mathrm{d}} \\
& \mathrm{p} 2=\mathrm{p}_{2 \Delta \mathrm{t}, \text { rev }}=\mathrm{p}_{\mathrm{a}, \mathrm{d}} \times \mathrm{p}_{\mathrm{d}, \mathrm{b}}=\mathrm{p}_{\mathrm{b}, \mathrm{d}} \times \mathrm{p}_{\mathrm{d}, \mathrm{a}}, \text { reverse probability during } 2 \text { time-steps. } \\
& \alpha=\text { learning coefficient } ; \alpha=.25 \\
& \lambda=\text { learning asymptote } \lambda=2 \cdot \mathrm{p} 1
\end{aligned}
$$

Empirical results:

$\begin{array}{lllllllll}\text { Period }(30 \mathrm{sec}): & 1 & 2 & 3 & 4 & 5 & 6 & 7 & 8 \\ \text { Reverses, means: } & \mathbf{2 . 5} & \mathbf{3 . 5} & \mathbf{3 . 8} & \mathbf{4 . 2} & \mathbf{4 . 3} & \mathbf{4 . 4} & \mathbf{4 . 6} & \mathbf{4 . 7}\end{array}$

Simulation results: The frequencies are means of 1000 simulations.

I) Time-step $0.02 \mathrm{sec}, \mathrm{p} 1=.0032, \mathrm{p} 2=.0016, \lambda=.0064$

$$
\begin{array}{lllllllll}
\text { Reverses, means: } & 2.73 & 3.38 & 3.86 & 4.24 & 4.47 & 4.64 & 4.68 & 4.79
\end{array}
$$

II) Time-step $0.5 \mathrm{sec}, \mathrm{p} 1=.08, \mathrm{p} 2=.04, \lambda=.16$

$$
\begin{array}{lllllllll}
\text { Reverses, means: } & 2.65 & 3.27 & 3.83 & 4.11 & 4.50 & 4.73 & 4.72 & 4.69
\end{array}
$$

Time-step: There are some psychological findings to use when we try to answer to the question of which time-step would be most realistic.

The starting point could be N. Whitehead's idea of the discreteness of consciousness: consciousness is composed of successive occasions of experience. Hameroff and Powell (2009) refer to this idea:

“... Alfred North Whitehead $(1929 ; 1933)$ viewed the universe as comprised not of things, but of events-in other words, as a process. ... Whitehead's occasions are spatio-temporal quanta, each endowed - usually at a very low level—with mentalistic characteristics like 'experience, subjective immediacy, appetition'." (p. 3). Further: "Our normal conscious experience seems continuous, but so does a motion picture-even though we know it to be composed of discrete frames. There is no doubt that we perceive motion pictures as continuous despite their actual 'quantum' structure. Perhaps consciousness is the same.” (p. 3).

Referring to Stroud's theory, Hameroff and Powell reason that the length of the occasion of experience needs to be $0.02-0.05 \mathrm{sec}$ :

“The 'perceptual moment' theory of Stroud described consciousness as discrete events, rather like sequential frames of a movie. Evidence in recent years suggests periodicities for perception and reaction times in the range of 20 to 50 milliseconds (gamma EEG waves; 30 to $90 \mathrm{~Hz}$ )..." (p. 3).

Case I above shows the simulation results when the length of the time-step is just $0.02 \mathrm{sec}(=20 \mathrm{msec})$. In this case $\mathrm{p} 1$ was .0032 . Thus, the transition probability matrix has the following form:

$\left.\begin{array}{lllll} & \multicolumn{5}{c}{\mathbf{M}_{\text {II }}} & & \\ & \mathrm{d} & \mathrm{a} & \mathrm{b} & \\ \mathbf{d} & \lceil 0 & .5 & .5 & \rceil \\ \mathbf{a} & .0032 & .9968 & 0 \\ \text { b } & \lfloor .0032 & 0 & .9968\end{array}\right]$

The matrix is, thus, the theoretical estimate for the figure-reverse process in the case of the Necker cube.

Note: Actually, we are quite free to choose the length of the time-step. In principle, there is no obstacle to using, e.g., Planck's time $\left(10^{-43} \mathrm{sec}\right)$ as the length of the time-step. In that case vector a would be: $\left(0,16 / 10^{43}, 1-\right.$ $\left.0,16 / 10^{43}, 0\right)$. The expectation value $r$ is computed by the equation: $r=t \cdot n_{\Delta t} \cdot p$, where $t=$ the length of the period in sec, $\mathrm{n}_{\Delta \mathrm{t}}=$ the number of time-steps per sec, and $\mathrm{p}=$ the probability of a reverse event during one time-step, $\mathrm{p}_{\mathrm{a}, \mathrm{d}}$ or $\mathrm{p}_{\mathrm{b}, \mathrm{d}}$. In our example, where we now use Planck's time as the time-step, the variables get these values:

$\mathrm{t}=30, \mathrm{n}_{\Delta \mathrm{t}}=10^{43}$, and $\mathrm{p}=0.08 / 10^{43}$. Thus, $\mathrm{r}=30 \cdot 10^{43} \cdot 0,08 / 10^{43}=30 \cdot 0.08=2.4$.

This result is rather a good estimate for the empirical frequency 2.5 .

The aim of our example concerning the reversible figures was to show that the DPM seems also to have estimation power, provided that suitable empirical data are in use. 


\subsection{The Usual Way of Gestalt Generation}

We develop further our analysis concerning the gestalt formation of reversible figures and examine the usual way of creating or selecting the gestalts.

There are different types of reversible figures: sometimes one alternative, $\mathbf{a}$, is dominant and another, $\mathbf{b}$, is rare. In that case the transition probability matrix is asymmetric and oblique, for example in the following way:

$\left.\begin{array}{llll} & & \mathbf{M}_{\text {II }} \\ & \mathrm{d} & \mathrm{a} & \mathrm{b} \\ \text { d } & \lceil .02 & .78 & .2\rceil \\ \text { a } & .01 & .99 & 0 \\ \text { b } & \lfloor .4 & 0 & .6\end{array}\right]$

where $\mathrm{d}=$ diffuse configuration, $\mathrm{a}=$ dominant gestalt, $\mathrm{b}=$ rare gestalt.

Going further in this direction, one can derive a model of "normal", i.e., extremely oblique gestalt generation, which is very common in our everyday routines and so dominant that it is nearly the only way of gestalt formation we recognize. In that "normal" case the matrix may have, for example, the following appearance:

\begin{tabular}{lllllll} 
& \multicolumn{7}{c}{$\mathbf{M}_{\text {II }}$} & & & & \\
& $\mathrm{d}$ & $\mathrm{a}$ & $\mathrm{b} 1$ & $\mathrm{~b} 2$ & $\mathrm{~b} 3$ & $\ldots$ \\
$\mathbf{d}$ & $\Gamma \approx 0$ & $\approx 1$ & $\approx 0$ & $\approx 0$ & $\approx 0 \ldots$ & \\
$\mathbf{a}$ & .01 & .99 & 0 & 0 & $0 \ldots$ & \\
$\mathbf{b 1}$ & .01 & 0 & .99 & 0 & $0 \ldots$ & \\
$\mathbf{b 2}$ & $\lfloor .01$ & 0 & 0 & .99 & $0 \ldots$ & $\ldots$
\end{tabular}

where $\mathrm{d}=$ diffuse configuration, $\mathrm{a}=$ dominant gestalt, $\mathrm{b} 1, \mathrm{~b} 2, \mathrm{~b} 3$, etc. $=$ rare ("abnormal") gestalts, $\approx 1=$ approximately 1 , and $\approx 0=$ approximately 0 .

The normal gestalt generation may be seen as a borderline case of the perception of reversible figures.

The new form of vector $\mathbf{d}$ of the matrix $\mathbf{M}_{\mathbf{I I}}$ can also be interpreted in such a way that the learning of the transition d-a has proceeded completely and reached its end.

The observable world which we try to interpret is, on every occasion, an extremely large group of perception alternatives like "reversible figures" and usually only one dominant gestalt is selected and interpreted as "real" in our consciousness.

\subsection{Gestalt's Further Differentiation. Mental Levels}

The gestalt formation of Rubin's figure (Fig. 3) does not remain in the superficial state of seeing some indefinite face profiles or the alternative, "a kind of vase". It can continue as a precise process and lead to a detailed differentiation: "Two relatively serious fellows with slightly curved noses". One has to notice that the perception is continuously the whole "two profiles" and that the "seriousness of the look" or the "curved nose" have their meanings only as characteristics of the whole. If the line of the "mouth" is separated from that, it would not be interpreted as a "mouth" at all. Thus, the detailed differentiation requires the figure to be observed for a long enough time, i.e., in terms of the DPM, the probability of remaining in the state of "two profiles" needs to be high enough and the time-step long enough. That means that the consciousness should have the chance to create autonomously a new system where the process has time to transit from the state of the diffuse configuration to one of the possible states of differentiated gestalts. We are inclined to speak in these cases of the different "levels" of consciousness (cf. David Bohm).

In Fig. 5, the levels of consciousness are illustrated schematically and the gestalt generation process of the reversible figure (Rubin's figure) is presented. At the first level, the process of the perception system reaches the state s which is called the state of invariant process of the perception contents. This is projected to the second level and appears there as the state $s$, which means the perception of a diffuse whole (without any verbal interpretation). At this second level, the gestalt generation process transits from the state $s$ to the state $p$ ("two diffuse profiles") or $v$ ("a diffuse vase"). The state $p$ at the second level may then be projected to the state $p$ at the third level. (Correspondingly, $v$ may be projected to $v$ at the third level.) Usually there is time enough for further gestalt generation: the process transits from $\mathrm{p}$ to $\mathrm{dp}$ at the third level (differentiated "two profiles" gestalt with 
verbal interpretation) or, correspondingly, from $\mathrm{v}$ to $\mathrm{dv}$ (differentiated "vase" gestalt). All the time there exist the conditions that the original perception process continues and the projections remain the same.

The differentiated gestalt ( $\mathrm{dp}$ or $\mathrm{dv}$ ) may be projected to a still higher level and the process may continue at that level, making the gestalt more differentiated in detail.

It is possible to analyze analogously the situations where the mental process has started spontaneously from a mental state at some high level of consciousness (imagination or thinking). The process may follow the same principles as the generation of perception gestalts examined above. This kind of analysis would, however, lead too far in the direction of wild speculation.

\section{Discussion}

This article is a "thought experiment" which was done only to show in a logical way how the dynamic phenomena of consciousness "look out" when they are analyzed according to quantum mechanics. Eccles' brilliant idea that between mental and physical phenomena there exists a connection that classical physics can not deal with is developed further in this article. It has been interpreted mathematically, to be precise in using the concept of interference between two quantum systems as the theoretical basis. (Interference is actually thought to be a consequence of entanglement between the two systems but it has to be emphasized that we have very little information about it.)

The solution of the mind/brain problem led us directly to the question of how physical brain states can produce mental states (experiences), i.e., how perception processes develop. It came out that in this case too the activity of the mental world needs to be taken as the starting point. Feedback between the synapses and the mental systems is absolutely essential, i.e., how the "experimentation procedure" of the mind becomes "reinforced" with "rewards" (success) or with "punishments" (failures).

This article may be only one attempt to analyze with quantum mechanics also the later stages of the perception process, the gestalt generation, although in the form of a rather speculative "thought experiment". In doing that, however, we found a particular case (reversible figures) where it was possible to build, according to the DPM, a model which gave really good estimates for empirical data. Thus, maybe the thinking throughout this article is not so speculative as it appears.

Many questions still remain totally open. This whole study is dependent on whether the concept of partial vector interference is accepted or not. There is not yet any empirical evidence for it and it has not been accepted into use in quantum mechanics. On the other hand, total vector interference is very clearly only a particular case of the more general partial vector interference (Rainio and Malaska, 2011).

Philosophically, a very central question concerning consciousness is the problem of the autonomy of mental systems. How do the systems come into being or be "produced"? Do some systems perhaps have a particular "ability to take into use" other systems? How much may the learning processes reduce the system's autonomy?

The extremely important question of the existence of free will concerns the problem of autonomy. It is not necessary in the frame of reference of DQM to exclude free will, although no conclusive evidence exists for its belonging to the systems' dynamics.

We also have to leave partially outside the scope of this article the question which Chalmers calls the "Hard Problem of Consciousness". What characteristics do the totally subjective experiences we call qualia have? Seemingly, they could be categorized as intrinsic properties of certain system states. This was partially done when the gestalt generation was assumed.

Chalmers actually presents the intrinsic properties of mental states as an explanation of experiences: "Physics requires information states but cares only about their relation, not their intrinsic nature: phenomenology requires information states but cares only about their intrinsic nature..." (Chalmers, 1996, p. 305).

Chalmers clearly differentiates the phenomenal aspects from the physical ones, according to the "two-aspect monism": "We might say that [the] internal aspects of these states are phenomenal and the external aspects are physical." (Chalmers, 1996, p. 305).

Chalmers emphasizes the internal characteristics of the phenomenal world: the experiences do not belong to the dynamical description of the systems (to the processes). Therefore they have no effect on the flow of the mental events. Thus, according to Chalmers, the phenomenal world remains epiphenomenal: it is not causally related to the physical reality because it does not control the mental dynamics.

To get out of this dilemma we have to take into account that there exist, seemingly, two qualities of intrinsic properties of mental states: 
a) Not all intrinsic properties are qualia-type. They may, in addition, participate in mental dynamics - differing from Chalmers' claim. For example, they are able to produce attraction, as well as value fields which are entangled in cognitive decision making (Lewin's "Life Space"; see Rainio 2008 and 2009b). Some value fields may be very extensive and long-lasting. The interferences of those kinds of value fields may dominate the dynamics of the psychic forces of an individual. In such cases the experiences really affect not only the mental processes but - through the synapses in the brain - indirectly, the physical world. It means that the mental causation needs to be taken as a true fact. Thus, we are able to avoid epiphenomenalism.

b) The intrinsic properties may be "pure" experiences without any dynamical qualities (without effects on the cognitive processes or decision making). For example, they may be pure qualia experiences. How those phenomena are related to the "reality" and how they can be examined (in additon to introspection) is the essential "hard problem".

There exists, thus, a partial solution to the "hard problem of consciousness". In some situations there is a causal relation of the experiences to the "reality", while in some situations only the subjective aspect is available.

If the quantum mechanical frame of reference appears to be fruitful and well argued in the analysis of consciousness and, particularly, if the research based on it acquires empiric evidence, then a change in the paradigm seems to be necessary. One has to give up materialistic monism and accept a new ontological viewpoint. We can keep a consistent, monistic ontological view but it cannot be materialistic any more, nor idealistic, but something we could call in-formation monism. (Writing in-formation with a hyphen is borrowed from David Bohm, who uses the word in its basic meaning: creating forms which control events. Bohm even strengthens this meaning by the concept active in-formation.) Some other thinkers support that view and are of the opinion that the whole of reality actually forms one comprehensive information system, e.g., Ervin Laszlo ("Science and the Akashic Field", 2004) and Mark Germine ("One Mind Model", Germine, 2010).

According to this new in-formation monism, the dynamics of events (the evolution of quantum systems) is controlled by in-formation fields, which are characteristically probability fields (transition probability matrices!). Matter and energy have their positions in this reality: they are special states of quantum processes; the laws of classical physics concern only these special states. It thus seems quite natural that they have no use in the analysis and description of conscious (mental) processes. In this context it may be suitable to quote Benjamin Libet, whose consciousness theory (CMF) has been described in the following way:

"Libet states that the CMF 'would not be a category of known physical fields, such as electromagnetic, gravitational, etc. The conscious mental field would be in a phenomenologically independent category; it is not describable in terms of any externally observable physical events or any known physical theory as presently constituted.'” (McFadden, 2000, p. 296, quotation from Libet, B.: Neurophysiology of Consciousness, 1993.)

That philosophical frame of reference which is called in-formation monism above is actually a modern version of an earlier ontological viewpoint called "neutral monism" (Spinoza, Russell etc.) and of the later "double-aspect monism" (Chalmers, Whitehead, Stapp etc.). In-formation monism goes deeper in ontology than double-aspect monism.

It is philosophically interesting to notice the radical change in the concept of causality during the development of paradigms in physics. Strict deterministic causality was very necessarily discarded when quantum mechanics required science to accept statistical causality, which means that only the distributions of variable values are causally determined. The examination of consciousness forces us to ask whether it would be plausible to claim that autonomous occurrences exist, which means that cases may exist where the causes of events may be totally lacking.

Seemingly, we are not able to create any theory of consciousness without taking the existence of autonomous systems into account. It should mean that the algorithmic modeling of the whole of reality is, even in principle, impossible (as, for example, Penrose has emphasized). There is, thus, no way to a "Theory of Everything" and no reasons to reduce the autonomy of such sciences as psychology.

\section{References}

Atmanspacher, H. (2004). Quantum Approaches to Consciousness. Stanford Encyclopedia of Philosophy. [Online] Available: http://plato.stanford.edu/entries/qt-consciousness/

Chalmers, D. J. (1996). The Conscious Mind: In Search of a Fundamental Theory, Oxford: Oxford University Press. 
Chalmers, D. J. (2006). Facing Up to the Problem of Consciousness. Journal of Consciousness Studies, 2(3):200-219, 1995. [Online] Available: http://consc.net/papers/facing.html

Eccles, J. C. (1994). How the Self Controls Its Brain. New York Berlin Heidelberg: Springer Verlag.

Germine, M. (2010). The Next Scientific Revolution: A New Theory of Mind, Evolution, and Quantum Reality Based on Process Metaphysics. Dynamical Psychology, 2010. [Online] Available: http://www.goertzel.org/dynapsyc/NextScientificRevolution.htm

Gudder, S. (1986). Discrete Quantum Mechanics, J. Math. Physics, 27, 1782 (1986). http://dx.doi.org/10.1063/1.527044

Hameroff, S., \& Powell, J. (2009): The Conscious Connection: A Psychophysical Bridge between Brain and Pan-experiential Quantum Geometry. In D. Skrbina, (Ed.): Mind That Abides: Panpsychism in the New Millennium. Amsterdam: Benjamins Pub. (Chapter 5).

Laszlo, E. (2004). Science and the Akashic Field; An Integral Theory of Everything. Rochester, Vermont: Inner Traditions.

McFadden, J. (2000): Quantum Evolution. London: HarperCollinsPublishers.

Ohta, N., \& MacLeod, C. M., \& Uttl, B. (Eds.). (2005): Dynamic Cognitive Processes. New York: Springer.

Rainio, K. (2008). Discrete Process Model for Quantum and Mind Systems. Research Reports 1/2008, Department of Social Psychology, Helsinki University. [Online] Available: http://ethesis.helsinki.fi/valspsjul.html

Rainio, K. (2009a). Discrete process model for quantum systems of matter and mind. World Futures, Vol. 65, 4, 2009, pp. 270-303. http://dx.doi.org/10.1080/02604020802392161

Rainio, K. (2009b). Kurt Lewin's Dynamical Psychology Revisited and Revised. Dynamical Psychology, 2009. [Online] Available: http://www.goertzel.org/dynapsyc/Rainio-Lewin's-psych-pdf-6-8-09.pdf

Rainio, K., \& Malaska, P. (2011). Vektori-interferenssi diskreetissä kvanttimekaniikassa (DQM). [Online] Available: http://www.lfs.fi/julkaisuja/verkkojulkaisuja

Revonsuo, A., \& Newman, J. (1999). Binding and consciousness. Consciousness and Cognition, 8 (2), 123-127, 1999. http://dx.doi.org/10.1006/ccog.1999.0393

Toppino, T.C., \& Long, G.M. (2005) Top-Down and Bottom-Up Processes in Perception of Reversible Figures. In: Ohta, N., \& MacLeod, C. M., \& Uttl, B. (Eds.): Dynamic Cognitive Processes. New York: Springer. http://dx.doi.org/10.1007/4-431-27431-6_3

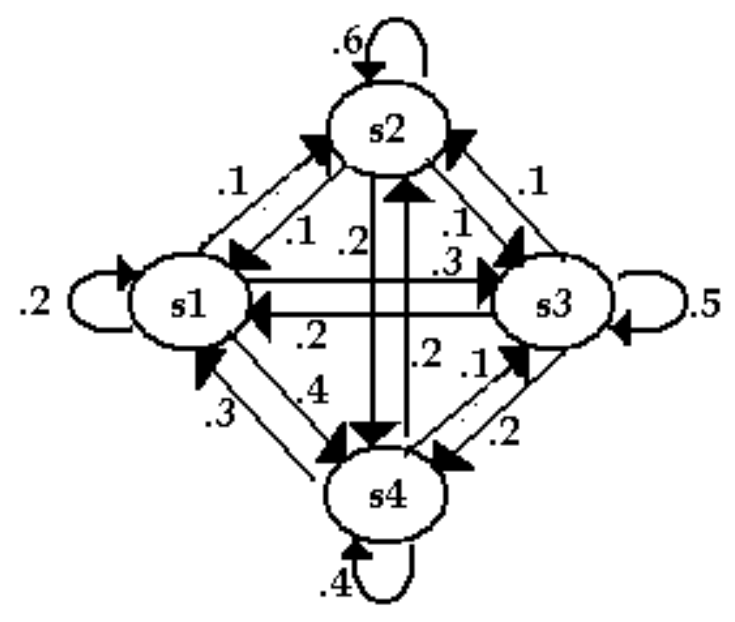

Figure 1. Transition probabilities in the form of a graph 


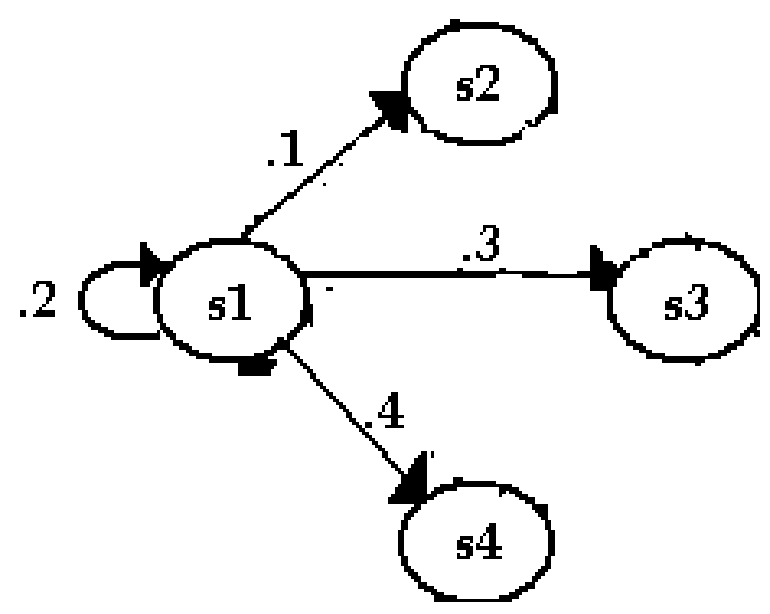

Figure 2. State vector in a graph form

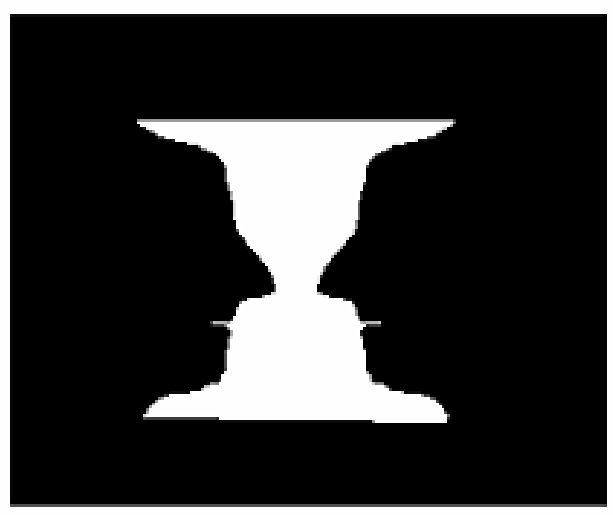

Figure 3. Rubin's figure

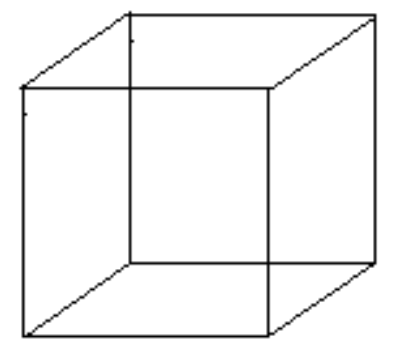

Figure 4. The Necker cube, a reversing figure.

Is the front square left and down (a) or right and up (b)? 


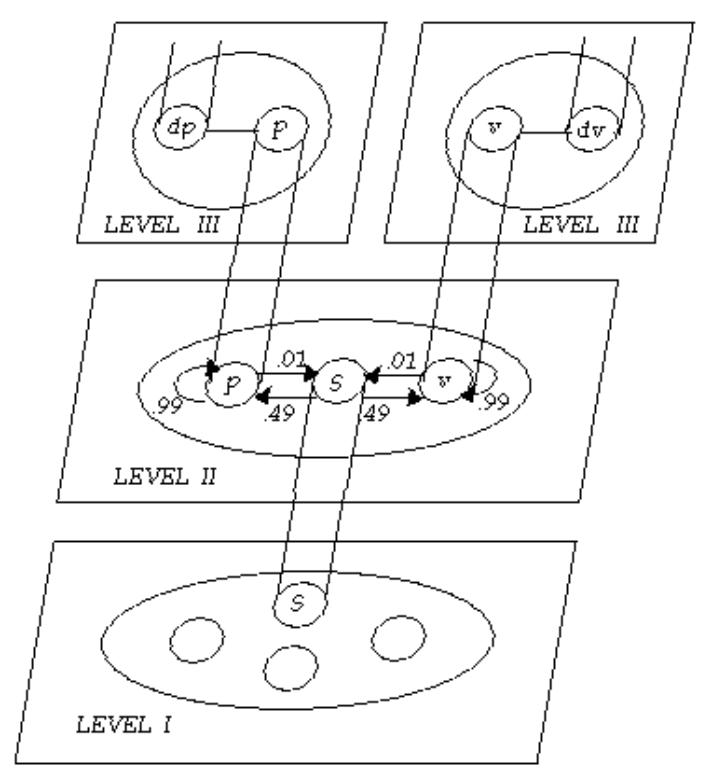

Figure 5. Levels of consciousness 